\title{
Lifetime cumulative number of menstrual cycles and serum sex hormone levels in postmenopausal women
}

\author{
Mariana Chavez-MacGregor • Carla H. van Gils • \\ Yvonne T. van der Schouw · Evelyn Monninkhof · \\ Paulus A. H. van Noord · Petra H. M. Peeters
}

Received: 13 March 2007/Accepted: 16 March 2007/Published online: 22 May 2007

(C) Springer Science+Business Media B.V. 2007

\begin{abstract}
Objective Lifetime cumulative number of menstrual cycles is related to breast cancer risk. The aim of this study is to investigate the relation between this index and serum sex hormone levels in postmenopausal women.

Methods Cross-sectional study including 860 naturally postmenopausal Dutch participants of the European Prospective Investigation into Cancer and Nutrition. Lifetime cumulative number of menstrual cycles was computed using questionnaire data on ages at menarche and menopause, number of pregnancies, breastfeeding, oral contraceptive use (OC) and regularity pattern. Measurements of hormones included estrone (E1), estradiol (E2), andostrenedione, testosterone, sex-hormone binding globulin (SHBG) and dehydroepiandrostenedione sulfate (DHEAS). The relation between the lifetime cumulative number of menstrual cycles and hormone levels was assessed using analysis of covariance. Relations between reproductive characteristics and hormone levels were also studied. Adjustments for characteristics at blood collection included age, years since menopause, BMI, hormone replacement therapy use, OC use, smoking habits, alcohol intake and physical activity were done.
\end{abstract}

M. Chavez-MacGregor - C. H. van Gils .

Y. T. van der Schouw . E. Monninkhof .

P. A. H. van Noord - P. H. M. Peeters

Julius Center for Health Sciences and Primary care, University Medical Center, STR 6.131, PO BOX 85500, 3508 GA Utrecht,

The Netherlands

M. Chavez-MacGregor $(\bowtie)$

Department of Medicine, Washington University School of

Medicine, Campus Box 8121, 660 South Euclid, St. Louis, MO

63110, USA

e-mail: mchavezm@im.wustl.edu
Results Lifetime cumulative number of cycles was related with SHBG; participants in the lowest category had higher SHBG levels. For the separate characteristics, DHEAS and androstenedione increased significantly with increasing age at menarche, while androstenedione and testosterone decreased with increasing age at menopause. For the parity characteristics, SHBG levels increased according to the number of live births.

Conclusions Lifetime cumulative number menstrual cycles was related only to SHBG. Therefore, free levels of estrogens or androgens may be related to this number of menstrual cycles estimate, reflecting lifetime exposure to ovarian hormones.

Keywords Reproductive Characteristics - Breast Cancer . Sex Hormones - Steroids - Cumulative number of menstrual cycles $\cdot$ Risk Factors

\section{Introduction}

Breast cancer is strongly related to various hormonal factors. We know that different hormones play a key role in the pathogenesis of invasive breast cancer in both, premenopausal and postmenopausal women [1-4], likely representing a direct effect of circulating hormones [5-7].

The patterns of risk associated with reproductive history suggest that a prolonged exposure to ovarian hormones increases breast cancer occurrence [8]. It has been well established that early age at menarche, late age at menopause, null parity and late age at first full term pregnancy (FFTP) are important risk factors for breast cancer [9-11]. These characteristics may have long lasting effects on hormone metabolism, although data regarding reproductive characteristics and hormone levels are still inconclusive [12-16] 
In an effort to measure the lifetime exposure to endogenous hormones, the concept of the "lifetime cumulative number of menstrual cycles" was introduced as an index that could reflect total ovarian exposure [17, 18]. The "lifetime cumulative number of cycles" summarizes information such as menarche, parity, menopause, regularity pattern, breast feeding and oral contraceptive (OC) use. In previous studies we, but also others, related the cumulative numbers of menstrual cycles to breast cancer risk [17, 18]. Ovarian function, and therefore, "lifetime number of menstrual cycles", is defined by age at menopause, and age at menopause is related to postmenopausal estrogen levels [19]; with this rationale we studied the association between the "lifetime number of menstrual cycles" and hormone levels after the menopause. The hypothesis fits in, where a common factor at hypothalamic level may affect the speed by which oocytes are being used, increasing postmenopausal gonoadothopin levels and maybe, stimulating ovarian testosterone release from the remaining theca cells, as well as non ovarian estrogens from adrenal origin.

The aim of this study was to evaluate the relation between the "lifetime cumulative number of menstrual cycles" and the reproductive characteristics that contribute to this estimate on one side, and plasma concentrations of sex hormones on the other side, in order to elucidate the mechanism by which reproductive risk factors could influence hormone-related cancers.

\section{Study design and methods}

The Prospect-EPIC study is one of the two Dutch cohorts participating in the European Prospective Investigation into Cancer and Nutrition; a multi-center prospective study carried out in 23 centers from 10 European countries [20].

A detailed description of the study population and data collection of the Prospect-EPIC study has been published elsewhere [21]. Briefly, 17,357 women aged 49-70, residing in Utrecht and its surroundings were recruited through a regional, population-based, breast cancer screening program between 1993 and 1997. Base-line characteristics were collected on the basis of two self-administered questionnaires (regarding lifestyle and nutrition). The lifestyle questionnaire contains information on demographic characteristics, family history, past and current morbidity, reproductive history (gynecologic and obstetric), smoking habits, and physical activity. In addition, anthropometric measurements (height, weight, and waist and hip circumference) were taken as well as a $30 \mathrm{ml}$ blood sample. Within $24 \mathrm{~h}$, samples of $4 \mathrm{ml}$ serum, $9 \mathrm{ml}$ citrate plasma, $2 \mathrm{ml}$ white blood cells, and $2 \mathrm{ml}$ red blood cells were fractionated into $0.5 \mathrm{ml}$ aliquots and stored at $-196^{\circ} \mathrm{C}$. Blood samples were successfully drawn from $97.5 \%$ of the participants [21]. All women signed a written informed consent and the study was approved by the Institutional Review Board of the University Medical Center Utrecht.

Of all Prospect-EPIC participants a $10 \%$ random sample $(n=1736)$ was taken and hormone measurements were done. For the present study, participants who used hormone replacement therapy (HRT) or OC in the previous twelve months were excluded $(n=311)$, as well as those women with history any cancer other than non-melanoma cancer $(n=25)$. From the remaining 1,400 participants only those who underwent natural menopause were eligible $(n=860)$ because only in them we had reliable information about ovarian activity not masked by the absence of menstrual cycles due to hysterectomy. Natural menopause was defined as the complete cessation of menstrual bleeding for 12 months preceding enrollment due to natural causes (according to self-report).

We computed the "lifetime cumulative number of menstrual cycles", of the women in this study. For parous women, the number of menstrual cycles before a FFTP was also estimated as we previously described [17]. Briefly, the "lifetime cumulative number of menstrual cycles" was computed from age at first menstrual period to age at menopause. Ages at menarche, at menopause and at FFTP were recorded as continuous variables. Age at regularization of cycles was computed according to the time between menarche and the establishment of regular periods (immediately after menarche, after 1, 2, 3, 5, or 10 years, after FFTP or never regular). The regularity pattern was defined categorically as 24, 26, 28, 30 and 32 day cycles, or always irregular. Information about OC use included: age at start, number of years of use and age at last use. Data about number of and age at each pregnancy, stillbirth, miscarriage and abortion, if any, were available, as well as the number of weeks of breastfeeding for each pregnancy.

We took all the previously mentioned variables into account when computing the "lifetime cumulative number of menstrual cycles', considering that each pregnancy, stillbirth, miscarriage or abortion, resulted in an absence of cycles for a period of 36, 28 and 12 weeks respectively. We assumed that lactation results in a 6-week absence of cycles [22]. Menstrual cycles due to OC use were computed as 28 days duration (see Appendix). Considering that irregular cycles are a combination of long and short menstrual periods, the number of irregular cycles was computed according to each participant's regularity pattern; the total number of irregular years was recorded in order to make further adjustments. The number of menstrual cycles was impossible to compute in the participants who reported to be always irregular or never having regular periods, therefore we added the "irregular' category as such in the analysis (See appendix). 
Bounded levels of estrogens and androgens were measured, levels of estrone (E1), estradiol (E2), androstenedione, testosterone, sex hormone binding globulin (SHBG) and dehydroepiandrostenedione sulfate (DHEAS) were measured in plasma using commercially available doubleantibody radioimmunoassay (RIA) kits (Diagnostic System Laboratories Inc., Webster, TX, USA). The following kits were used: E1: DSL-8700, E2: DSL-39100, androstenedione: DSL-4200, TST: DSL-4100 SHBG: DSL-6300 and DHEAS: DSL-2700. The intra-assay coefficients of variation were $5.6 \%, 3.9 \%, 4.3 \%, 7.7 \%, 3.0 \%$ and $5.2 \%$ respectively. The inter-assay coefficients of variation were $11.1 \%, 4.1 \%, 6.3 \%, 8.1 \%, 4.0 \%$ and $5.3 \%$ respectively. All hormone assays were performed by the laboratory of the Hormones and Cancer group at the International Agency for Research on Caner (IARC). Although technically SHBG is not a hormone, for reasons of convenience it will be referred as such. We calculated the free-androgen index (FAI) dividing testosterone by SHBG and multiplying by 100 , the SHGB/E2 index was calculated dividing SHBG by E2.

\section{Data analysis}

Means with their standard deviation (SD) values, or frequencies of baseline characteristics were calculated. Concentrations of all hormones were logarithmically transformed to produce approximately normal distributions. To make the results easily interpretable, we transformed the mean and its 95\% confidence interval (CI) back to their original scale, resulting in geometric means and 95\% CIs. Geometric mean levels and 95\% CIs were calculated for each hormone (SHBG, E1, E2, androstenedione, testosterone, DHEAS), SHBG/estradiol index (SHBG/ E2) and free androgen index (FAI).

Using analysis of covariance models, geometric mean hormone levels were calculated for the different categories of cumulative number of menstrual cycles (lifetime and before a FFTP). The same analysis was also used for categories of age at menarche, age at menopause, parity, number of children and age of FFTP. In order to build the adjusted model we classified confounders in three categories as follows: Group A contained characteristics clearly related to hormone levels such as age, years since menopause and body mass index (BMI); Group B contained factors possibly related to hormone levels like OC and HRT use, smoking habits, physical activity and alcohol consumption; group $\mathrm{C}$ contained characteristics unlikely related to hormone levels such as marital status, education level and family history of breast cancer. We first adjusted for all confounders of type $\mathrm{A}, \mathrm{B}$, and $\mathrm{C}$, then we built models by deleting $\mathrm{B}$ and $\mathrm{C}$ types of characteristics, without affecting validity but increasing precision. In the final model we included confounders in group A and B, therefore we adjusted for age at intake as a continuous variable, number of years from menopause to inclusion in the study, BMI at examination, use of HRT ever $(\mathrm{Y} / \mathrm{N})$, use of OC ever $(\mathrm{Y} / \mathrm{N})$, smoking habits, alcohol consumption and physical activity. Information on current alcohol use was obtained from the food frequency questionnaire, total alcohol consumption in grams per day was calculated from questions in the number of glasses of wine, beer, fortified wines, and liquor/spirits per day, week, month or year. One glass of any alcoholic beverage was assumed to contain $10 \mathrm{~g}$ of alcohol. With the daily physical activity data from the general questionnaire, the Voorrips score was calculated (and was entered in the model as a continuous variable), this score has been developed and validated especially for women in this age group [23]. For analysis of the "lifetime cumulative number of menstrual cycles" and the "number of cycles before a FFTP"' variables, we also adjusted for more than one year of irregular cycles $(\mathrm{Y} / \mathrm{N})$. Given that age at menopause can be determined using age at intake in combination of years since menopause; when analyzing age at menopause, the covariate years since menopause was not entered in the model.

To test the significance of the models' linearity, median values in each category for every variable were computed accordingly. Subsequently these values were included as a continuous variable in a linear regression model to test for linear trends. Because there is no clear natural hierarchy, the "always irregular" category was not included when testing for trend for the "lifetime number of menstrual cycles" and the "number of menstrual cycles before a FFTP" variables. Additional mutual adjustments for reproductive characteristics did not show any difference in the observed trends. The Statistical Package for Social Sciences for WINDOWS, version 14.0 (Chicago, IL), was used for all statistical analysis, and all tests were two sided.

\section{Results}

General and reproductive characteristics of the ProspectEPIC women that were included in this study are shown on Table 1. On average, participants were 60.2 years old at enrollment; had a BMI of $26.2 \mathrm{~kg} / \mathrm{m}^{2}$ and the majority of them $(69.4 \%)$ had at least low technical-secondary education. $113(13.1 \%)$ were nulliparous, and for the parous, the mean age for a FFTP was 25.5 years old. Mean age at menarche and menopause were 13.6 and 49.6 years old respectively, the time from menopause to inclusion, was on average 10.6 years. In 786 participants $(91.4 \%)$ the lifetime cumulative number of cycles was calculated, as we had incomplete data regarding reproductive history in 74 women. The median number of lifetime menstrual cycles was 
Table 1 General and reproductive characteristics of the study participants $(n=860)$

\begin{tabular}{|c|c|}
\hline & Mean (SD) \\
\hline \multicolumn{2}{|l|}{ General characteristics } \\
\hline Age at enrollment & $60.2(5.5)$ \\
\hline BMI & $26.2(4.2)$ \\
\hline Waist Hip ratio & $0.797(0.59$ \\
\hline Length (cm) & $163.7(6.1)$ \\
\hline Weight (Kg) & $70.1(11.9)$ \\
\hline \multicolumn{2}{|l|}{ Educational level (\%) } \\
\hline - Primary education & $263(30.6)$ \\
\hline - Low technical, secondary & $384(44.6)$ \\
\hline - High technical, secondary & $98(11.4)$ \\
\hline - Academic & $115(13.4)$ \\
\hline Ever married (\%) & $805(93.6)$ \\
\hline \multicolumn{2}{|l|}{ Cigarette smoking habits $(\%)$} \\
\hline - Never smoked & $402(46.7)$ \\
\hline - Past smoker & $275(32)$ \\
\hline - Current smoker & $183(21.3)$ \\
\hline Mother or sister with BC (\%) & $123(14.3)$ \\
\hline \multicolumn{2}{|l|}{ Reproduction characteristics } \\
\hline Age at menarche & $13.6(1.6)$ \\
\hline Nulliparous (\%) & $113(13.1)$ \\
\hline Age at FFTP $^{\mathrm{a}}$ & $25.46(3.8)$ \\
\hline Number live born children ${ }^{\mathrm{a}}$ & $2.9(1.4)$ \\
\hline Age at menopause & $49.6(4.0)$ \\
\hline Years since menopause & $10.6(6.0)$ \\
\hline Oral contraceptive use $(\%)$ & $384(44.6)$ \\
\hline Years of OC use ${ }^{b}$ & 6 \\
\hline HRT use $(\%)$ & $66(7.7)$ \\
\hline Years of HRT use ${ }^{c}$ & 1 \\
\hline \multicolumn{2}{|c|}{ Lifetime number of menstrual cycles } \\
\hline$(n=786)$ & $165(21.0)$ \\
\hline$\leq 415$ & $177(22.5)$ \\
\hline $416-453$ & $171(21.8)$ \\
\hline $454-490$ & $161(20.5)$ \\
\hline$\geq 491$ & $112(14.2)$ \\
\hline
\end{tabular}

Number of menstrual cycles before

FFTP $(n=683)$

$\leq 118$

$167(25.5)$

$119-147$

$160(23.4)$

$148-185$

$118(17.3)$

$\geq 186$

$143(20.9)$

Always irregular ${ }^{\mathrm{d}}$

95 (13.9)

\footnotetext{
a Among parous women

b Median, Among OC users

c Median, Among HRT users

d Participants in the always irregular category, reported to be never regular
}

451.3, corresponding to 34.7 years of menstrual activity if considering an average of 13 cycles per year. From the 747 parous participants, 64 had incomplete data to compute the number of menstrual cycles before a FFT, in the remaining 683 participants $(91.4 \%)$, the median number of menstrual cycles before a FFTP was 146.7 (approximately 11.3 years of menstrual activity). These results are consistent with the previous data on such estimates for a larger sample of the Prospect-EPIC participants as we previously published [17]. Table 2 shows the geometric mean hormone levels for all participants with 95\% CIs of SHBG, E1, E2, androstenedione, testosterone, DHEAS, SHBG/E2 and FAI.

Table 3 shows the unadjusted geometric mean hormone levels and 95\% CI for each hormone according to some general and reproductive characteristics. When testing for trend participants in the lower quartile of "lifetime cumulative of menstrual cycles" had higher levels of SHBG, androstenedione, testosterone and DHEAS than the participants with the highest quartile ( $P$ trend $=0.015$, $0.003,0.052$ and 0.035 respectively), and lower levels of SHBG/E2 $(P$ trend $=0.031)$.

In table 4 adjusted geometric means for each hormone according to the reproductive characteristics are shown. Participants in the lowest quartile of cumulative number of menstrual cycles tended to have higher SHBG ( $P$ trend $=0.013)$ and lower SHBG/E2 $(P$ trend $=0.050)$ levels. Androstenedione and DHEAS levels increased with increasing age at menarche $(P$ trend $=0.027$ and 0.022 respectively), a similar tendency was observed for E2, although the $P$ for trend did not reach statistical significance $(P$ trend $=.058)$. Women with more children, tended to have higher SBHG levels $(P$ trend $=0.030)$, number of children was also related to lower SHBG/E2 ( $p$ trend $=0.010$ ) levels. No association was found between number of cycles before a FFTP, parity, age at FFTP and any of the hormones. Age at menopause related inversely with androstenedione and testosterone; women with older

Table 2 Geometric mean serum sex hormone and SHBG levels

\begin{tabular}{ll}
\hline Hormone & Mean, $(95 \% \mathrm{CI})$ \\
\hline SHBG $\mu \mathrm{g} / \mathrm{ml}$ & $18.1(17.2-19.2)$ \\
Estrone $\mathrm{pg} / \mathrm{ml}$ & $15.2(14.48-16.0)$ \\
Estradiol $\mathrm{pg} / \mathrm{ml}$ & $8.5(8.2-8.8)$ \\
Androstenedione $\mathrm{ng} / \mathrm{ml}$ & $0.47(0.45-0.49)$ \\
Testosterone total $\mathrm{ng} / \mathrm{ml}$ & $0.26(0.25-0.27)$ \\
DHEAS ng/ml & $455.1(432.9-478.4)$ \\
SHBG- Estradiol index $(\mu \mathrm{g} / \mathrm{pg})$ & $0.47(0.44-0.50)$ \\
Free androgen index & $4.6(4.4-5.0)$ \\
$\quad[($ Testosterone $/ \mathrm{SHBG} * 100(\mathrm{ng} / \mu \mathrm{g} * 100)]$ & \\
\hline
\end{tabular}




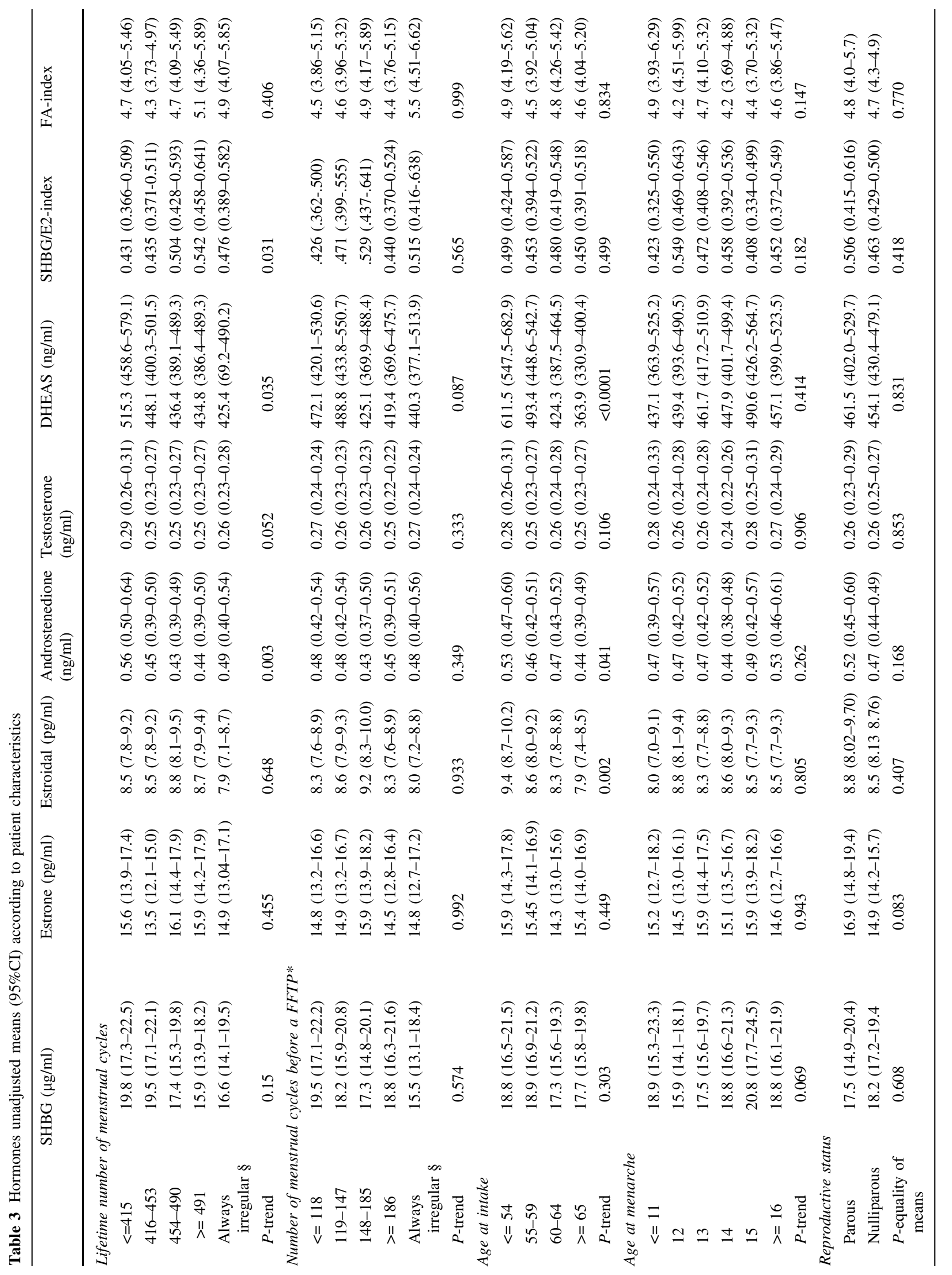




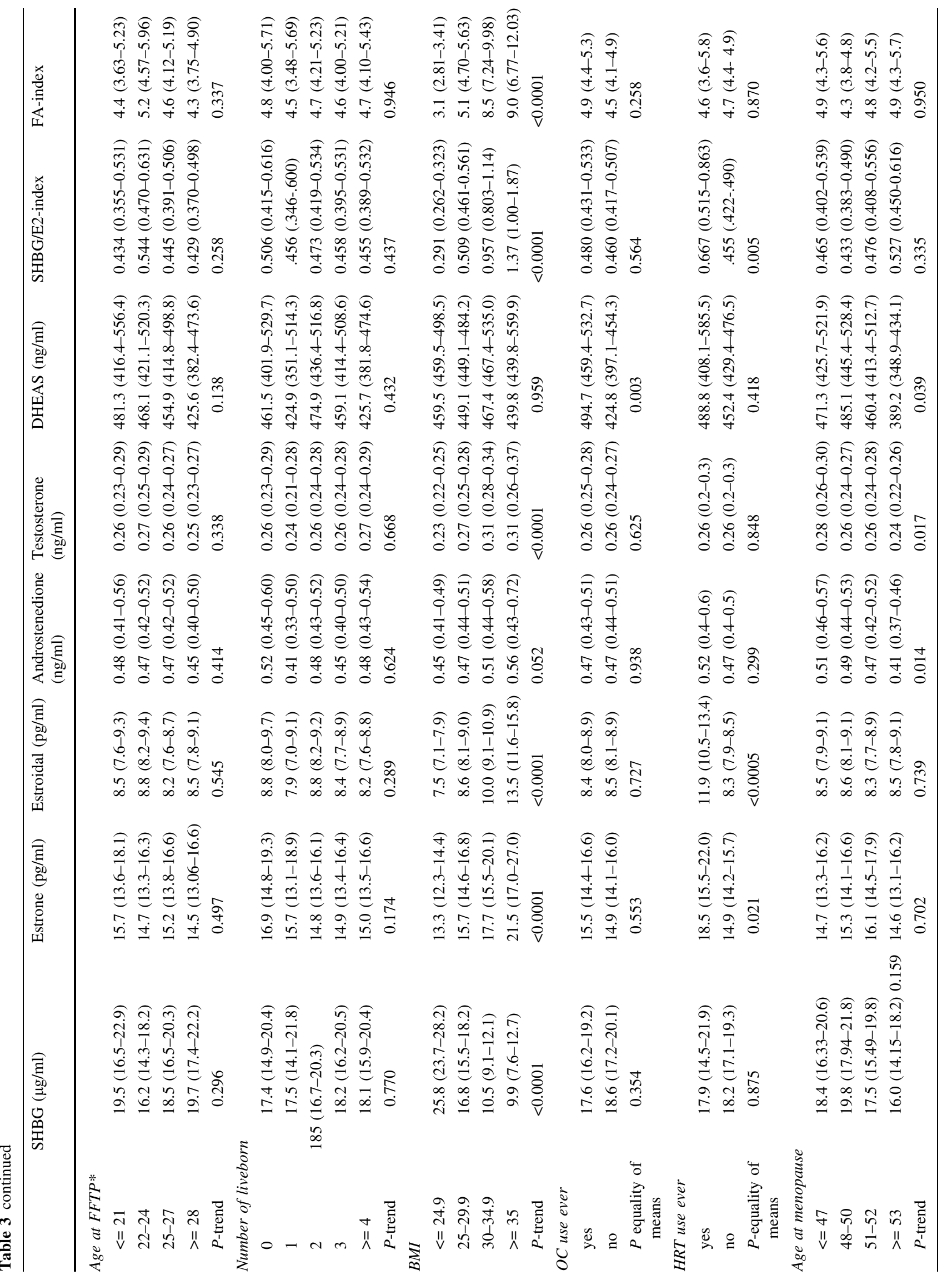


age at menopause tend to have lower levels of such hormones ( $p$ trend $=0.024$ and 0.015 respectively).

\section{Discussion}

The results of the present study suggest that the "lifetime cumulative number of cycles" is related to SHBG and that some reproductive characteristics are also associated to postmenopausal serum sex hormone levels. We noted significant associations after adjusting for important confounders, the "lifetime cumulative number of menstrual cycles" related inversely with levels of SHBG and positively with $\mathrm{SHBG} / \mathrm{E} 2$. Age at menarche directly relates to both DHEAS and androstenedione. Number of children had a significant relation with levels of SHBG and SHBG/ E2 and age at menopause was indirectly associated to androstenedione and testosterone.

Several studies have investigated the relation between reproductive risk factors and endogenous sex hormone levels in postmenopausal women, but with conflicting results $[6,13,15,24-27]$. The patterns of risk associated with reproductive history suggest that prolonged exposure to ovarian hormones increases breast cancer occurrence [8, 28, 29]. The cumulative number of menstrual cycles has been used as a proxy for ovarian activity, women in the highest quartile of the "lifetime cumulative number of menstrual cycles", estimate, have an increased breast cancer risk when compared with women in the lowest quartile [17, 18]. A significant inverse relation between number of menstrual cycles and SHBG was observed in our study. It has been previously described that SHBG decrease the proportion of E2 that is able to leave the circulation and enter the cells, and an inverse relation between concentration of SHBG and breast cancer has been observed in epidemiological studies [1, 10]. Our findings suggest therefore, that postmenopausal free levels of estrogens or androgens may be related to the "lifetime cumulative number of menstrual cycles" reflecting lifetime exposure to ovarian hormones.

Early age at menarche and late age at menopause have long being recognized as risk factors for breast cancer. A 1year delay in the onset of menarche is associated with a 5\% reduction in risk for developing breast cancer in later life [30]. We found that age at menarche has a direct relation with serum levels of DHEAS and androstenedione measured after the menopause. Androgens have been associated with breast cancer risk, women with breast cancer tend to have higher levels of DHEAS and androstenedione [2, 4, $31,32]$. It has been proposed that in postmenopausal women DHEAS might act as a moderate estrogen agonist, through competitive binding of its metabolite 5-androstene-3B,17B-diol [33]. It is also well known that the 


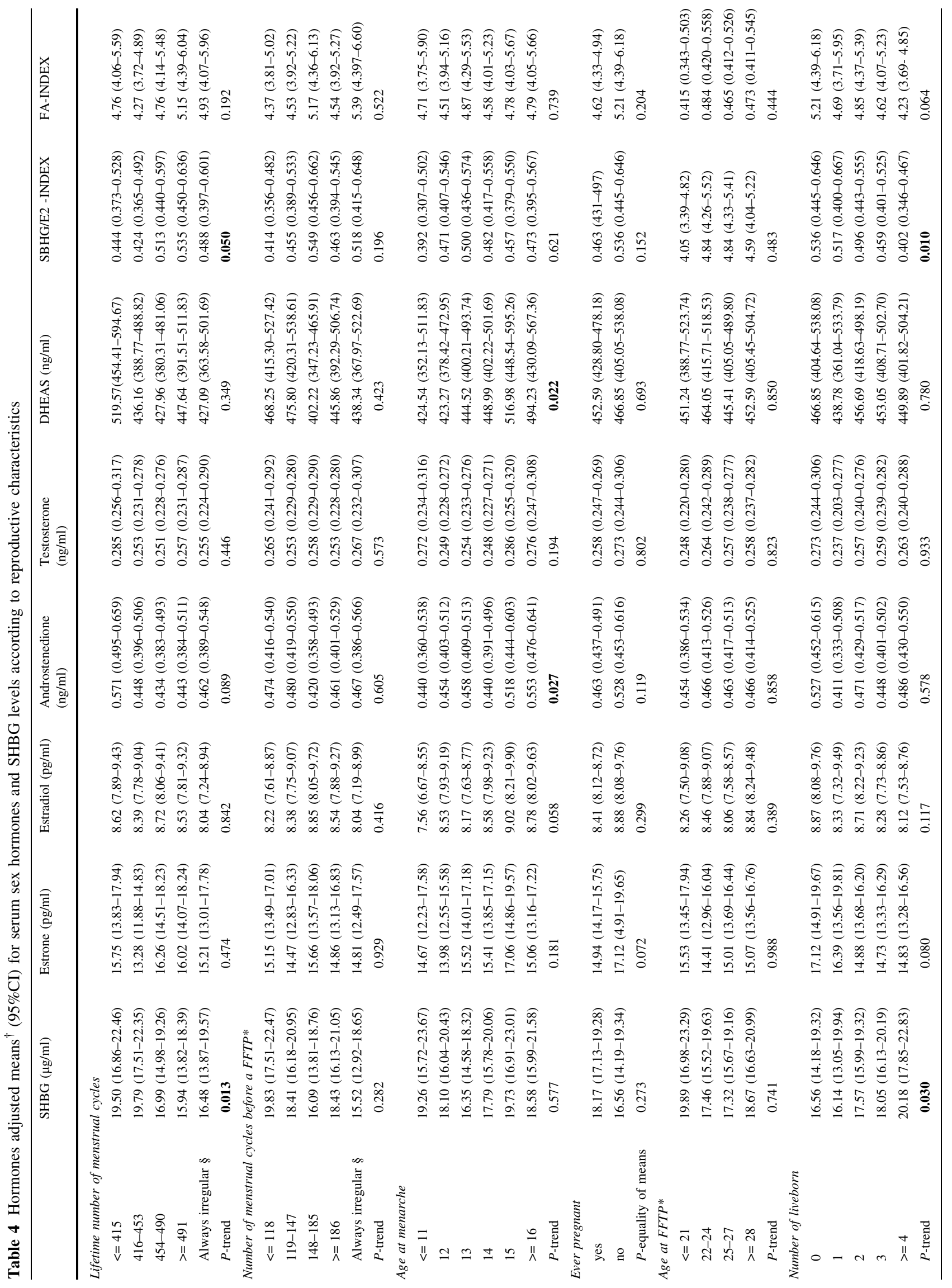


peripheral conversion of adrenal androstenedione is the principal source of E1 in postmenopausal women [34], therefore we would expect an inverse association between both DHEAS and androstenedione and age at menarche. Madigan et al. showed, after adjusting for BMI and years since menopause, that young age at menarche, was associated with higher levels of androstenedione, but also of E1 and E2 [15]. Our results are in conflict with such findings, however several studies have found no association between age at menarche and SHBG, estrogens or androgens [14, $16,35,36]$.

Childbearing and a young age at FFTP protect against breast cancer and additional pregnancies further reduce the risk $[7,10,37,38]$. We found that parous women tend to have lower levels of E1, however this difference was not statistically significant $(P=.072)$. Lamar et al. [14] found that nulliparous women had higher testosterone levels, a finding that could contribute to the increased risk of breast cancer in nulliparous women. Our findings also showed that the number of live births is directly related to SHBG, and indirectly to SHBG/E2 and FAI $(P$ for trend for FAI did not reach statistical significance, $P=0.064$ ). This observation is in conflict with the known association between low SBGH levels and breast cancer risk, and the protective effect that the number of pregnancies has on breast cancer [28, 29, 38, 39]. It is important to mention that a woman's genetic and environmental profile may influence both, her ability to have children and her postmenopausal sex hormone concentrations, making these observations difficult to interpret. It has been hypothesized that the overall effect of parity is due to a protective effect of a young age at FFTP [40], another proposed mechanism is through lactation, as breastfeeding induces final differentiation of the terminal duct epithelium making it relatively insensitive to hormonal stimulation [41, 42]. Despite these observations we found no relation between age at FFTP and any of the hormones. No relation between parity, number of pregnancies or age at FFTP and serum hormone levels was reported by several studies. [12, 14-16, 43, 44].

We observed an indirect association between age at menopause and both, androstenedione and testosterone. Several studies have failed in finding an association between age at menopause and sex hormones [12, 14, 16], however a direct association with menopause and androstenedione has been reported [15], as well as a relation between age at menopause and E2 in a group of 173 postmenopausal overweight women [13].

To appreciate the findings of our study, some strengths and limitations need to be mentioned. The main advantage is our large sample size, to the best of our knowledge this is the largest study assessing the relationship between reproductive risk factors and serum sex hormone levels in 
naturally menopausal women and the first one evaluating the relationship between sex hormones and an index that can be used as a proxy of ovarian exposure. Another advantage is that we attempted to adjust for all relevant covariates, in order to obtain fully adjusted estimates. The participants in this cross-sectional study originated from a random sample of a large cohort, and blood samples where drawn from $97.5 \%$ of the 17,357 participants, favoring the generalization of our results. Given the prospective nature of the Prospect-EPIC study, and because participants were recruited through an existing population-based breast cancer screening program, we included only disease-free participants at baseline blood collection.

A limitation of this study is that only a single blood sample was assayed per participant, however it has been shown that a single measure provides substantial information in postmenopausal women for E1, E2 and SHBG [45]. Serial measurements of serum hormones in postmenopausal women suggest that a single measurement can reliably categorize women, at least over the short term. Intraclass correlation coefficients of hormone measurements in serum collected over a 3-year period were reported to be between 0.68 and 0.78 for the estrogens, 0.66 and 0.88 for the androgens, and 0.92 for SHBG [46]. We did not have information regarding premenopausal sex hormone concentrations in the participants of this study; it remains unknown whether some underlying hormonal profile could have influenced both, reproductive capabilities and postmenopausal sex hormone concentrations. It is also possible that premenopausal hormone levels are more related to reproductive factors than postmenopausal levels, and that is as a proxy for premenopausal levels that postmenopausal levels relate to reproductive characteristics. We should consider that the data used regarding reproductive characteristics are prone to recall errors. However, it is unlikely that recall errors are related to endogenous hormone levels themselves, if any misclassification occurred, it would have been nondifferential.

In summary, in this group of naturally postmenopausal women, the "lifetime cumulative number menstrual cycles" was related only to SHBG, suggesting that free levels of estrogens or androgens may be related to this index, reflecting lifetime exposure to ovarian hormones. Our findings show that some reproductive characteristics are related to postmenopausal serum sex hormone levels, however we do not find clear evidence to explain the mechanism by which reproductive risk factors influence hormone-related cancers in naturally postmenopausal women. It seems that reproductive and menstrual characteristics do not affect postmenopausal breast cancer risk via a long-term effect on sex hormone levels. The association of reproductive risk factors and breast cancer could be a result of prolonged exposure to ovarian hormones, and not only the product of a direct, long-lasting effect of sex hormone serum concentrations. Further research is needed in order to unravel the biological mechanisms through which reproductive characteristics relate to breast cancer risk.

Acknowledgements This project was financed by the European Commission-Europe Against Cancer: WHO AEP/90/05; the Dutch Ministry of Health; the Dutch Prevention Funds and the Dutch Heart Foundation (Grant 99-159). Mariana Chavez-Mac Gregor was receiving the merit-based academic CONACYT scholarship in support of postgraduate education (from the National Council for Science and Technology, Mexico).The authors declare that there is no conflict of interest that would prejudice the impartiality of this scientific work.

\section{Appendix}

This is a simple example of a woman with an age at menarche of 12 years, one miscarriage at 23 , an age at FFTP of 25 and 2 children who she breastfeed 10 weeks and 7 weeks. This participant also reported to have regular periods since her menarche with cycles every 30 days, OC use during 5 years starting at age 29 , and having menopause at age 51. Her number of menstrual cycles until her FFTP is 147 , and the number of menstrual cycles during her lifetime is 452.8 .

Number of cycles until FFTP: 25 years at FFTP-12 years at menarche $=13$ years. 13 years $\times$ 52.178 weeks in one year $=678.314$ weeks in 13 years - (12 weeks miscarriage at 23 )-(36 weeks first pregnancy) $=630.31$ weeks $=4412.198$ days. 4412.198 days $/$ $30=147.07$ cycles ( 30 days duration).

Lifetime number of menstrual cycles: 51 years at menopause -12 years at menarche $=39 \quad(-5$ years OC use $=34) . \quad 34$ years $\times 52.178$ weeks in one year $=1774.071$ weeks in 34 years- $(12$ weeks of miscarriage) - $36 \times 2$ weeks of two pregnancies) —(17 weeks total breastfeeding $)-(6 \times 2$ weeks absence of cycles after lactation $)=1661.071$ weeks $=11627.5$ days.

11627.5 days $/ 30=387.58$ cycles ( 30 days duration).

5 years of OC use $\times 365.25=1461$ days in 5 years, $1826.25 / 28=65.22$ cycles $(28$ days duration due to OC use. $387.58+65.22=452.8$ menstrual cycles during lifetime.

\section{References}

1. The Endogenous Hormones and Breast Cancer Collaborative Group (2002) Endogenous sex hormones and breast cancer in postmenopausal women: reanalysis of nine prospective studies. $\mathbf{J}$ Natl Cancer Inst 56(8):606-616

2. Kaaks R, Berrino F, Key T, Rinaldi S, et al (2005) Serum sex steroids in premenopausal women and breast cancer risk within the European prospective investigation into cancer and nutrition (EPIC). J Natl Cancer Inst 97(10):755-765 
3. Kaaks R, Rinaldi S, Key TJ, et al (2005) Postmenopausal serum androgens, oestrogens and breast cancer risk: the European prospective investigation into cancer and nutrition. Endocr Relat Cancer 12(4):1071-1082

4. Eliassen AH, Missmer SA, Tworoger SS, et al (2006) Endogenous steroid hormone concentrations and risk of breast cancer among premenopausal women. J Natl Cancer Inst 98(19):14061415

5. Onland-Moret NC, Kaaks R, Van Noord PA, et al (2003) Urinary endogenous sex hormone levels and the risk of postmenopausal breast cancer. Br J Cancer 88(9):1394-1399

6. Moore JW, Key TJ, Bulbrook RD, Clark GM, Allen DS, Wang DY, Pike MC (1987) Sex hormone binding globulin and risk factors for breast cancer in a population of normal women who had never used exogenous sex hormones. Br J Cancer 56(5):661666

7. Key TJ, Verkasalo PK (1999) Endogenous hormones and the aetiology of breast cancer. Breast Cancer Res 1(1):18-21

8. Henderson BE, Ross RK, Judd HL, et al (1985) Do regular ovulatory cycles increase breast cancer risk?. Cancer 56(5):12061208

9. Hulka BS, Moorman PG (2001) Breast cancer: hormones and other risk factors. Maturitas 38(1):103-113

10. Travis RC, Key TJ (2003) Oestrogen exposure and breast cancer risk. Breast Cancer Res 5(5):239-247

11. Key TJ, Verkasalo PK, Banks E (2001) Epidemiology of breast cancer. Lancet Oncol 2(3):133-140

12. Boyapati SM, Shu XO, Gao YT, (2004). Correlation of blood sex steroid hormones with body size, body fat distribution, and other known risk factors for breast cancer in post-menopausal Chinese women. Cancer Causes Control 15(3):305-311

13. Chubak J, Tworoger SS, Yasui Y, et al (2004) Associations between reproductive and menstrual factors and postmenopausal sex hormone concentrations. Cancer Epidemiol Biomarkers Prev 13(8):1296-1301

14. Lamar CA, Dorgan JF, Longcope C, et al (2003) Serum sex hormones and breast cancer risk factors in postmenopausal women. Cancer Epidemiol Biomarkers Prev 12(4):380-383

15. Madigan MP, Troisi R, Potischman N, et al (1998) Serum hormone levels in relation to reproductive and lifestyle factors in postmenopausal women (United States). Cancer Causes Control 9(2):199-207

16. Verkasalo PK, Thomas HV, Appleby PN, et al (2001) Circulating levels of sex hormones and their relation to risk factors for breast cancer: a cross-sectional study in 1092 pre- and postmenopausal women (United Kingdom). Cancer Causes Control 12(1):47-59

17. Chavez-MacGregor M, Elias SG, Onland-Moret NC, et al (2005) Postmenopausal breast cancer risk and cumulative number of menstrual cycles. Cancer Epidemiol Biomarkers Prev 14(4):799_ 804

18. Clavel-Chapelon F (2002) Cumulative number of menstrual cycles and breast cancer risk: results from the $\mathrm{E} 3 \mathrm{~N}$ cohort study of French women. Cancer Causes Control 13(9):831-838

19. Zeleniuch-Jacquotte A, Shore RE, Koenig KL, et al (2004) Postmenopausal levels of oestrogen, androgen, and SHBG and breast cancer: long-term results of a prospective study. $\mathrm{Br} \mathrm{J}$ Cancer 90(1):153-159

20. Riboli E, Hunt KJ, Slimani N, et al (2002) European Prospective Investigation into Cancer and Nutrition (EPIC): study populations and data collection. Public Health Nutr 5(6B):1113-1124

21. Boker LK, van Noord PA, van der Schouw YT, et al (2001) Prospect-EPIC Utrecht: study design and characteristics of the cohort population. European prospective investigation into cancer and nutrition. Eur J Epidemiol 17(11):1047-1053

22. Cunningham FG, Gant NF, Leveno KJ et al (eds) (2001) Williams Obstetrics, 21st edn. McGraw-Hill, USA
23. Voorrips LE, Ravelli AC, Dongelmans PC, et al (1991) A physical activity questionnaire for the elderly. Med Sci Sports Exerc 23(8):974-979

24. Dorgan JF, Reichman ME, Judd JT, et al (1995) Relationships of age and reproductive characteristics with plasma estrogens and androgens in premenopausal women. Cancer Epidemiol Biomarkers Prev 4(4):381-386

25. Hankinson SE, Colditz GA, Hunter DJ, et al (1995) Reproductive factors and family history of breast cancer in relation to plasma estrogen and prolactin levels in postmenopausal women in the Nurses' Health Study (United States). Cancer Causes Control 6(3):217-224

26. Nagata C, Kabuto M, Takatsuka N, et al (1997) Associations of alcohol, height, and reproductive factors with serum hormone concentrations in postmenopausal Japanese women. Steroid hormones in Japanese postmenopausal women. Breast Cancer Res Treat 44(3):235-241

27. Newcomb PA, Klein R, Klein BE, et al (1995) Association of dietary and life-style factors with sex hormones in postmenopausal women. Epidemiology 6(3):318-321

28. Parazzini F, La Vecchia C, Negri E, et al (1993) Lifelong menstrual pattern and risk of breast cancer. Oncology 50(4):222-225

29. Rautalahti M, Albanes D, Virtamo J, et al (1993) Lifetime menstrual activity-indicator of breast cancer risk. Eur J Epidemiol 9(1):17-25

30. Hunter DJ, Spiegelman D, Adami HO, et al (1997) Non-dietary factors as risk factors for breast cancer, and as effect modifiers of the association of fat intake and risk of breast cancer. Cancer Causes Control 8(1):49-56

31. Colditz GA (2005) Epidemiology and prevention of breast cancer. Cancer Epidemiol Biomarkers Prev 14(4):768-772

32. Wang DY, Allen DS, De Stavola BL, et al (2000) Urinary androgens and breast cancer risk: results from a long-term prospective study based in Guernsey. Br J Cancer 82(9):1577-1584

33. Ebeling P, Koivisto VA (1994) Physiological importance of dehydroepiandrosterone. Lancet 343(8911):1479-1481

34. Cauley JA, Gutai JP, Kuller LH, et al (1989) The epidemiology of serum sex hormones in postmenopausal women. Am J Epidemiol 129(6):1120-1131

35. Bernstein L (2002) Epidemiology of endocrine-related risk factors for breast cancer. J Mammary Gland Biol Neoplasia 7(1):315

36. Bernstein L, Pike MC, Ross RK, et al (1991) Age at menarche and estrogen concentrations of adult women. Cancer Causes Control 2(4):221-225

37. de Waard F (1991) Endocrine aspects of cancer: an epidemiological approach. J Steroid Biochem Mol Biol 40(1-3):15-19

38. MacMahon B, Cole P, Lin MT, et al (1970) Age at first birth and cancer of the breast. A summary od an international study. Bull WHO 43:209-221

39. Choi NW, Howe GR, Miller AB, et al (1978) An epidemiologic study of breast cancer. Am J Epidemiol 107(6):510-521

40. de Waard F (1998) Risk factors for breast cancer at various ages. Eur J Cancer Prev 7(Suppl 1):S13-S15

41. Russo J, Russo IH (1999) Cellular basis of breast cancer susceptibility. Oncol Res 11(4):169-178

42. Newcomb PA, Storer BE, Longnecker MP, et al (1994) Lactation and a reduced risk of premenopausal breast cancer. N Engl J Med 330(2):81-87

43. Dorgan JF, Reichman ME, Judd JT, Brown C, et al (1995) Relationships of age and reproductive characteristics with plasma estrogens and androgens in premenopausal women. Cancer Epidemiol Biomarkers Prev 4(4):381-386

44. Nagata C, Kabuto M, Takatsuka N, et al (1997) Associations of alcohol, height, and reproductive factors with serum hormone concentrations in postmenopausal Japanese women. Steroid 
hormones in Japanese postmenopausal women. Breast Cancer Res Treat 44(3):235-241

45. Key TJ (1999) Serum oestradiol and breast cancer risk. Endocr Relat Cancer 6(2):175-180
46. Hankinson SE, Manson JE, Spiegelman D, et al (1995) Reproducibility of plasma hormone levels in postmenopausal women over a 2-3-year period. Cancer Epidemiol Biomarkers Prev 4(6):649-654 